\title{
EMISSÕES OTOACÚSTICAS EVOCADAS POR ESTÍMULO TRANSIENTE: PROTOCOLOS DE TRIAGEM AUDITIVA NEONATAL
}

\section{Transient evoked otoacoustic emissions (teoe): newborn hearing screening program protocols}

\author{
Isabela Freixo Côrtes-Andrade (1), Daniela Veronese Bento ${ }^{(2)}$, Doris Ruthi Lewis ${ }^{(3)}$
}

\begin{abstract}
RESUMO
Objetivo: descrever os resultados de três protocolos de EOAET de um equipamento automático utilizado em um Programa de TAN. Método: o equipamento utilizado foi programado com três critérios diferenciados de passa/falha. Protocolo A: Passar em 4 bandas de frequência, não precisando ser consecutivas; Protocolo B: Passar em 3 bandas de frequência, não precisando ser consecutivas e Protocolo C: Passar em 2 bandas de frequência, não precisando ser consecutivas. Os parâmetros para considerar presença de respostas foram: reprodutibilidade acima de $50 \%$, relação sinal ruído $>$ que $3 \mathrm{~dB}$ em 1.0 e $1.5 \mathrm{kHz}$ e > 6dB em 2.0, 3.0 e $4.0 \mathrm{kHz}$. Resultados: 574 orelhas foram analisadas, sendo que as bandas de frequência de $2.0,3.0$ e $4.0 \mathrm{kHz}$ foram as que apresentaram percentuais de "passa" mais elevados (94,1; 95,8 e 92,7\%, respectivamente). A banda de frequência de $1.0 \mathrm{kHz}$ obteve resposta presente em apenas $9,9 \%$ das orelhas testadas. Verificou-se que a análise do Protocolo C (2 bandas) foi a que apresentou o maior percentual de passa (96,9\%). Porém este percentual não é considerado estatisticamente diferente do Protocolo $B, 3$ bandas, $(96,2 \%)$ com valor de $p=0,520$. Assim, ambas as bandas são consideradas iguais, podendo-se utilizar qualquer uma das duas. Entretanto o Protocolo A (4 bandas) obteve 79,8\% de percentual de passa, apresentando uma taxa de falha elevada $(22,2 \%)$. Conclusões: deve-se pesquisar, com o padrão-ouro, todos os protocolos para verificação daquele com melhor sensibilidade e especificidade, apesar de terem apresentado resultados similares entre critérios de passa para 2 ou 3 bandas de frequência.
\end{abstract}

DESCRITORES: Audição; Perda Auditiva; Triagem Neonatal; Recém-Nascido

\section{INTRODUÇÃO}

O diagnóstico da deficiência auditiva, nos primeiros seis meses de vida, é um fator decisivo para minimizar os efeitos irreversíveis que a privação sensorial pode acarretar no desenvolvimento global $^{1-3}$. Nesse período crítico de maturação

(1) Fonoaudióloga; Doutoranda do Programa de Estudos PósGraduados em Fonoaudiologia da PUC-SP; Mestre do Programa de Estudos Pós-Graduados em Fonoaudiologia da PUC-SP.

(2) Fonoaudióloga; Mestranda do Programa de Estudos PósGraduados em Fonoaudiologia da PUC-SP.

(3) Fonoaudióloga; Professora Titular do Programa de PósGraduação em Fonoaudiologia e Faculdade de Fonoaudiologia da PUC-SP; Doutora em Saúde Pública pela USP.

Conflito de interesses: inexistente neurológica, tem-se o início do desenvolvimento auditivo quando ocorre uma prontidão para que as habilidades perceptivas básicas e a linguagem possam ser adquiridas ${ }^{4-6}$.

A prevalência da perda auditiva neurossensorial severa ou profunda varia de quatro a seis para cada 1000 nascidos vivos ${ }^{7}$, ou de um a três em 1000, conforme o Comitê Brasileiro sobre Perdas Auditivas $^{8,9}$. Esses achados mostram que, em determinadas populações, esta porcentagem aumenta drasticamente, como é o caso de crianças que permanecem em Unidades de Terapia Intensiva Neonatal (UTI-N), cuja prevalência encontrada é de $10,2 \%{ }^{10,11}$.

Atualmente, a implantação dos Programas de Triagem Auditiva Neonatal Universal (PTANU) está cada vez mais comum em todos os países ${ }^{3}$. No 
Brasil, em 02 de agosto de 2010, foi decretada e sancionada a Lei oㅜ 12.1303, que torna obrigatória a realização das Emissões Otoacústicas Evocadas nos neonatos, em todas as maternidades.

Em virtude desta demanda, Programas e Comitês Multiprofissionais vêm sendo desenvolvidos com objetivo de discutir e referendar ações voltadas à saúde auditiva de neonatos e lactentes, como o Joint Committee of Infant Hearing $(\mathrm{JClH})$, nos EUA; e o COMUSA, no Brasil ${ }^{12-14}$.

A implantação da TANU tem como objetivo identificar alterações auditivas que podem comprometer o desenvolvimento adequado das habilidades comunicativas de todas as crianças ao nascimento ou, no máximo, até os três meses de idade. Em caso de confirmação de comprometimento auditivo, é necessário que seja realizada a intervenção precocemente até os seis meses de idade, como recomenda o $\mathrm{JCIH}^{13}$. Deste modo, a implantação de um programa de TANU só tem sentido quando é realizado o diagnóstico para caracterizar o status auditivo, seguido da intervenção adequada para potencializar o desenvolvimento das habilidades auditivas e comunicativas ao longo do desenvolvimento ${ }^{12}$.

Autores sugerem que a realização da Triagem Auditiva Neonatal utilize as medidas eletroacústicas e eletrofisiológicas, como as Emissões Otoacústicas Evocadas por Estímulo Transiente (EOAET) e o Potencial Evocado Auditivo de Tronco Encefálico Auditivo Automático (PEATE-A), uma vez que essa população é incapaz de responder adequadamente a testes comportamentais devido à idade ${ }^{15-20}$. Essas duas técnicas têm sido largamente aplicadas como instrumentos eficientes na Triagem Auditiva Neonatal Universal (TANU), tanto em países desenvolvidos quanto em países em desenvolvimento'.

As EOAET tornaram-se um dos principais instrumentos para a detecção de alterações auditivas de origem coclear, pois possibilitam o estudo de aspectos mecânicos da função coclear de forma não-invasiva e objetiva e, independe do potencial de ação neural ${ }^{21}$, possibilitam a obtenção de informações objetivas, clinicamente, sobre os elementos pré-neurais da cóclea.

Este método não quantifica a deficiência auditiva, porém detecta sua presença ${ }^{21}$ em virtude de as EOAET estarem presentes em todos os indivíduos cujos limiares auditivos estão melhores que $20 \mathrm{e}$ $30 \mathrm{dBnNA}$. Assim sendo, a presença desse fenômeno pode confirmar a integridade do mecanismo coclear, podendo estabelecer a funcionalidade da atividade otoacústica das Células Ciliadas Externas (CCE) da cóclea. Em razão dessas características, além da rapidez e fidedignidade, esse exame tem 0 perfil ideal para os PTAN ${ }^{22,23}$.
O National Institutes of Health $(\mathrm{NIH})$ realizou uma pesquisa com o intuito de determinar as características e o desempenho de três exames que avaliam o sistema auditivo periférico: EOAET, EOAE-PD e o PEATE. Nesse estudo, 7.179 neonatos foram testados quanto à presença de cinco bandas de frequências $(1,0,1,5,2.0,3.0$ e $4.0 \mathrm{kHz})$. Com base nos resultados encontrados, foram recomendados os parâmetros de análise que garantem mais confiabilidade ao exame. Detectou-se que as EOAET são afetadas dependendo da frequência, pois os níveis de ruído são maiores quando as frequências testadas são mais baixas, tornando-se mais difícil de individuar a presença de uma emissão otoacústica evocada com espectro abaixo de $1,5 \mathrm{kHz}^{24}$.

Diferentes parâmetros e critérios de passa/ falha são utilizados nos equipamentos automáticos, os quais podem modificar a sensibilidade e especificidade do teste aplicado a uma população de neonatos e lactentes. No entanto é necessário que se escolham critérios seguros para que falso-positivos e falso-negativos não ocorram em números excessivos ou permitidos, dessa maneira, mantendo a confiabilidade deste exame. Nessa perspectiva, o objetivo do presente estudo foi descrever os resultados de três protocolos de EOAET, analisando 5 bandas de frequência de EOAET, de um equipamento automático utilizado em um Programa de Triagem Auditiva Neonatal.

\section{MÉTODO}

Foram estudados os resultados das EOAET de 287 neonatos em uma maternidade pública da cidade de São Paulo, entre os meses de abril a junho de 2010.

As triagens foram realizadas por alunos do Programa de Estudos Pós-Graduados em Fonoaudiologia da PUC-SP (mestrado e doutorado), além de fonoaudiólogas voluntárias. A equipe foi dividida em escalas durante a semana para garantir 0 plantão diário, exceto aos domingos, dia em que a TAN não é realizada.

Preferencialmente, as triagens foram realizadas próximas à alta hospitalar, entre 48 horas de vida, em uma sala silenciosa ou no próprio leito, com o bebê em sono natural. Não foi realizada a TAN em recém-nascidos com menos de 24 horas de vida ${ }^{25}$.

O Programa de Triagem Auditiva Neonatal (PTAN) utilizou como protocolo a pesquisa das Emissões Otoacústicas Evocadas Transientes (EOAET) em neonatos, sem Indicadores de Risco para Deficiência Auditiva (IRDA); e o Potencial Evocado Auditivo de Tronco Encefálico Automático (PEATE-A) para identificar comprometimentos auditivos nos neonatos com IRDA. 
Para concretizar o objetivo do presente estudo somente foram analisados os resultados de passa e falha das EOAET. Para o registro das EOAET foi utilizado o equipamento Otoport Lite da marca Otodynamics, que usa o estímulo clique nãolinear, que ocorre predominantemente na faixa de frequências entre 1.000 a $4.000 \mathrm{~Hz}$, com intensidade de $64 \mathrm{~dB}$ pe NPS. O critério de análise das emissões foi relação sinal/ruído de $6 \mathrm{~dB}$ SNR em 4 de 5 bandas de frequência testadas. Foram utilizados 260 sweeps de 16 estímulos apresentados, o tempo de teste máximo foi de 300 segundos; e as bandas de frequência de registro do aparelho foram: $1,01.5,2.0,3.0$ e $4.0 \mathrm{kHz}$. Os parâmetros utilizados para considerar presença de respostas foram: reprodutibilidade acima de $50 \%$, bem como relação sinal ruído > que $3 \mathrm{~dB}$ em 1.0 e $1.5 \mathrm{kHz}$ e > $6 \mathrm{~dB}$ em 2.0, 3.0 e $4.0 \mathrm{kHz}$.

Foram pesquisados três protocolos diferentes para permitir a análise de critérios de passa/falha distintos. Além dessa possibilidade, foi possível visualizar o resultado final de passa/falha para cada uma das cinco bandas de frequência testadas.

- Protocolo A: Passa-Falha em quatro bandas de frequência;
- Protocolo B: Passa-Falha em três bandas de frequência;

- Protocolo C: Passa-Falha em duas bandas de frequência.

O estudo foi aprovado pelo Comitê de Ética em Pesquisa da Pontifícia Universidade Católica de São Paulo - PUC/SP, com Protocolo de Pesquisa no063/2010.

Os resultados obtidos foram organizados, em uma planilha Excel, para a realização da análise estatística quantitativa por meio do teste de Igualdade de Proporções para comparação dos resultados, além do P-valor.

\section{RESULTADOS}

Os resultados para cada frequência e para as bandas de frequência em cada orelha foram analisados por meio do teste de Igualdade de Duas Proporções (Tabela 1 e Tabela 2). Somente na análise de 4 bandas foi encontrada uma pequena e significante diferença na distribuição das duas orelhas. No entanto, em todos os demais resultados, as orelhas comportaram-se de forma igual.

Tabela 1 - Distribuição das orelhas para cada frequência

\begin{tabular}{|c|c|c|c|c|c|c|}
\hline \multirow{2}{*}{\multicolumn{2}{|c|}{ Frequência }} & \multicolumn{2}{|c|}{ Orelha Direita } & \multicolumn{2}{|c|}{ Orelha Esquerda } & \multirow{2}{*}{ p-valor } \\
\hline & & $\mathbf{N}$ & $\%$ & $\mathbf{N}$ & $\%$ & \\
\hline \multirow{2}{*}{$1 \mathrm{kHz}$} & Passa & 24 & $8,4 \%$ & 33 & $11,5 \%$ & 0,209 \\
\hline & Falha & 263 & $91,6 \%$ & 254 & $88,5 \%$ & \\
\hline \multirow{2}{*}{$1,5 \mathrm{kHz}$} & Passa & 223 & $77,7 \%$ & 229 & $79,8 \%$ & \multirow{2}{*}{0,540} \\
\hline & Falha & 64 & $22,3 \%$ & 58 & $20,2 \%$ & \\
\hline \multirow{2}{*}{$2 \mathrm{kHz}$} & Passa & 265 & $92,3 \%$ & 275 & $95,8 \%$ & \multirow{2}{*}{0,077} \\
\hline & Falha & 22 & $7,7 \%$ & 12 & $4,2 \%$ & \\
\hline \multirow{2}{*}{$3 \mathrm{kHz}$} & Passa & 272 & $94,8 \%$ & 278 & $96,9 \%$ & \multirow{2}{*}{0,211} \\
\hline & Falha & 15 & $5,2 \%$ & 9 & $3,1 \%$ & \\
\hline \multirow{2}{*}{$4 \mathrm{kHz}$} & Passa & 262 & $91,3 \%$ & 270 & $94,1 \%$ & \multirow{2}{*}{0,200} \\
\hline & Falha & 25 & $8,7 \%$ & 17 & $5,9 \%$ & \\
\hline
\end{tabular}

Tabela 2 - Distribuição das orelhas para cada banda de frequência

\begin{tabular}{|c|c|c|c|c|c|c|}
\hline \multirow{2}{*}{\multicolumn{2}{|c|}{ Banda }} & \multicolumn{2}{|c|}{ Orelha Direita } & \multicolumn{2}{|c|}{ Orelha Esquerda } & \multirow{2}{*}{ p-valor } \\
\hline & & $\mathbf{N}$ & $\%$ & $\mathbf{N}$ & $\%$ & \\
\hline \multirow{2}{*}{4 Bandas } & Passa & 218 & $76,0 \%$ & 240 & $83,6 \%$ & \multirow{2}{*}{0,022} \\
\hline & Falha & 69 & $24,0 \%$ & 47 & $16,4 \%$ & \\
\hline \multirow{2}{*}{3 Bandas } & Passa & 272 & $94,8 \%$ & 280 & $97,6 \%$ & \multirow{2}{*}{0,082} \\
\hline & Falha & 15 & $5,2 \%$ & 7 & $2,4 \%$ & \\
\hline \multirow{2}{*}{2 Bandas } & Passa & 275 & $95,8 \%$ & 281 & $97,9 \%$ & \multirow{2}{*}{0,151} \\
\hline & Falha & 12 & $4,2 \%$ & 6 & $2,1 \%$ & \\
\hline
\end{tabular}


Tendo em vista que os resultados das orelhas direita e esquerda não obtiveram diferenças estatisticamente significantes, o tamanho da amostra foi dobrado e, assim, analisadas 574 orelhas com o intuito de deixar o estudo ainda mais fidedigno.

$\mathrm{Na}$ comparação das 5 frequências para as respostas de passa e falha, também, foi utilizado o teste de Igualdade de Duas Proporções (Tabela 3 e Tabela 4). Na tabela 3, é possível observar a distribuição proporcional entre todos os níveis de resposta. Já, na Tabela 4, estão os p-valores. Assim, verificou-se que a frequência com maior percentual de passa foi a $3 \mathrm{kHz}$ com $95,8 \%$, e que, consequentemente, teve o menor percentual de falha, com apenas $4,2 \%$. Porém, na análise de p-valores (Tabela 4), nota-se que esta frequência não pode ser considerada estatisticamente diferente da frequência de $2 \mathrm{kHz}$, com $94,1 \%$ de passa e $5,9 \%$ de falha.

Tabela 3 - Distribuição das frequências

\begin{tabular}{ccccc}
\hline \multirow{2}{*}{ Frequência } & \multicolumn{2}{c}{ Passa } & \multicolumn{2}{c}{ Falha } \\
\cline { 2 - 5 } & $\mathbf{N}$ & $\%$ & $\mathbf{N}$ & $\%$ \\
\hline $1 \mathrm{kHz}$ & 57 & $9,9 \%$ & 517 & $90,1 \%$ \\
\hline $1,5 \mathrm{kHz}$ & 452 & $78,7 \%$ & 122 & $21,3 \%$ \\
\hline $2 \mathrm{kHz}$ & 540 & $94,1 \%$ & 34 & $5,9 \%$ \\
\hline $3 \mathrm{kHz}$ & 550 & $95,8 \%$ & 24 & $4,2 \%$ \\
\hline $4 \mathrm{kHz}$ & 532 & $92,7 \%$ & 42 & $7,3 \%$ \\
\hline
\end{tabular}

Tabela 4 - P-valores da tabela 3

\begin{tabular}{ccccc}
\hline & $1 \mathrm{kHz}$ & $1,5 \mathrm{kHz}$ & $2 \mathrm{kHz}$ & $3 \mathrm{kHz}$ \\
\hline $1,5 \mathrm{kHz}$ & $<0,001$ & & & \\
\hline $2 \mathrm{kHz}$ & $<0,001$ & $<0,001$ & & \\
\hline $3 \mathrm{kHz}$ & $<0,001$ & $<0,001$ & 0,178 & \\
\hline $4 \mathrm{kHz}$ & $<0,001$ & $<0,001$ & 0,342 & $\mathbf{0 , 0 2 2}$ \\
\hline
\end{tabular}

Na comparação dos três protocolos pelos quais foram analisadas as bandas de frequência para as respostas de passa/falha (Tabela 5), foi possível verificar que a banda com o maior percentual de passa foi a que considerou 2 bandas de frequência, Protocolo C, com 96,9\%. Porém, estatisticamente, este percentual não é considerado diferente dos $96,2 \%$ da análise de 3 bandas, Protocolo B ( $p$-valor $=0,520$ ) (Tabela 6). Por conseguinte, ambas as bandas de frequência são consideradas iguais na distribuição de passa/falha. Entretanto o Protocolo
A (4 bandas) obteve $79,8 \%$ de percentual de passa, apresentando uma taxa de falha elevada $(22,2 \%)$ ao se comparar com os Protocolos B $(3,8 \%)$ e C $(3,1 \%)$.

Tabela 5 - Distribuição das bandas

\begin{tabular}{ccccc}
\hline Bandas de & \multicolumn{2}{c}{ Passa } & \multicolumn{2}{c}{ Falha } \\
\cline { 2 - 5 } Frequência & $\mathbf{N}$ & $\%$ & $\mathbf{N}$ & $\%$ \\
\hline 4 Bandas & 458 & $79,8 \%$ & 116 & $20,2 \%$ \\
\hline 3 Bandas & 552 & $96,2 \%$ & 22 & $3,8 \%$ \\
\hline 2 Bandas & 556 & $96,9 \%$ & 18 & $3,1 \%$ \\
\hline
\end{tabular}

Tabela 6 - P-valores da tabela 5

\begin{tabular}{lcc}
\hline & 4 Bandas & 3 Bandas \\
\hline 3 Bandas & $<0,001$ & \\
\hline 2 Bandas & $<0,001$ & 0,520 \\
\hline
\end{tabular}

\section{DISCUSSÃO}

Em razão de não haver diferença significante na análise estatística das EOAET quanto à orelha direita e esquerda (Tabela 1 e Tabela 2), a discussão deste estudo enfatiza, apenas, os resultados em que foram analisadas as duas orelhas simultaneamente.

Desta forma, as medidas de respostas de EOAET mais robustas (definidas pela relação sinalruído - SNR) foram observadas para as frequências de 3, 2 e 4kHz, conforme observado nas Tabelas 3 e 4. Estes resultados corroboram com o estudo que verificou níveis de emissões mais elevados para as mesmas frequências encontradas ${ }^{26}$.

Ademais, verificou-se que a frequência com menor percentual de passa foi a de $1 \mathrm{kHz}$, com $9,9 \%$, que, consequentemente, teve o maior percentual de falha, com $90,1 \%$. O alto índice de falhas nas Emissões Otoacústicas (EOA) é atribuído a muitos fatores, como ambientes ruidosos e ruídos fisiológicos que podem interferir no registro das EOAET, especialmente, nas faixas de frequências mais baixas ${ }^{26}$. Casos de falso-positivos na TAN, também, têm sido associados, principalmente, aos efeitos dos ruídos internos e externos ${ }^{26}$. Os ruídos internos são gerados pelo próprio sujeito (tosse, deglutição, ronco, respiração), já as fontes externas incluem o ruído do ambiente e outras interferências eletromagnéticas ${ }^{27}$.

Levando em consideração que o tempo de teste máximo do aparelho automático para registrar as 
EOAET, no PTAN do presente estudo, é de 300 segundos, para obter resultados presentes em frequências mais baixas, seria necessário dedicar um tempo de ensaio maior. Dada a SNR pobre e aumento do tempo dedicado à medida, a utilidade dos esforços para obter informações de $1,0 \mathrm{kHz}$ e $1.5 \mathrm{kHz}$, como parte de um programa de TANU, não é necessária. No entanto o ruído ambiental ainda pode contaminar o registro das respostas de EOAET e causar resultados falsos, especialmente, quando sons contínuos ou reverberantes estão presentes. Dessa forma, o problema de ruído, nas baixas freqüências, não é completamente resolvido com o uso dos filtros de controle ${ }^{27}$.

Como o sinal das EOA tem amplitude reduzida, a qual, normalmente, encontra-se entre -20 e 20dBNPS, o ruído ambiental é, comumente, a causa mais importante de problemas na avaliação das $\mathrm{EOA}^{28}$. Em razão do ruído ambiental, torna-se difícil determinar se as EOA estão ausentes pelo não-funcionamento dos processos ativos da cóclea ou pelo nível de ruído ser simplesmente elevado ${ }^{23}$. Pesquisadores não recomendam incluir como rotina as medições nas frequências de $1,0 \mathrm{kHz}$ e $1,5 \mathrm{kHz}$, sugerindo que os programas de TANU utilizem como critérios de passa as frequências mais altas ${ }^{29}$.

Ademais, verificou-se que a distribuição de passa e falha, comparando-se os três diferentes protocolos programados no equipamento automático de EOAET e levando em consideração a distribuição das bandas de frequência, na análise do Protocolo C (2 bandas), deram-se índices de presença de resposta mais elevados, com $96,9 \%$. No entanto este percentual não é considerado estatisticamente diferente do Protocolo B, que considerou a presença de 3 bandas $(96,2 \%)$, pois este apresentou valor de $p=0,520$. Sendo assim, ambas as bandas podem ser consideradas semelhantes na distribuição de passa/falha, podendo as duas ser utilizadas como protocolos de Programas de TANU.

O Protocolo A, que considerou a presença de 4 bandas, apontou $79,8 \%$ de percentual de passa, apresentando uma taxa de falha elevada, com $22,2 \%$, maior índice de falso-positivo, quando comparado aos Protocolos B $(3,8 \%)$ e C $(3,1 \%)$. Esses índices remetem a pensar nos casos de falso-positivos, ou seja, que apresentam resultado alterado devido a outros fatores que não estão relacionados com acometimentos orgânicos do sistema auditivo. Deve-se evitar casos de falso-positivos, pois podem gerar problemas na qualidade do programa, criando ansiedade desnecessária nos pais $^{16}$, além de aumentar os encaminhamentos ao reteste das falhas das EOAET e sobrecarregar os ambulatórios responsáveis pelo diagnóstico auditivo ${ }^{14}$.
A literatura descreve alguns estudos nacionais e internacionais utilizando critérios de passa e falha diferentes, indicando que ainda não existe um consenso atual de qual seria o melhor critério de passa/falha que o fonoaudiólogo deveria adotar utilizando as emissões otoacústicas evocadas transientes na triagem auditiva neonatal. Em 2000, um estudo, com o intuito de garantir a qualidade dos exames neonatais, utilizou como protocolo de passa/falha critério mais rigoroso, relação sinalruído (SNR) presente em quatro das cinco bandas testadas $(1.0,1.5,2.0,3.0 \text { e } 4.0 \mathrm{~Hz})^{26}$. Outras pesquisas não descrevem o motivo da escolha, porém encontram-se, como resultado satisfatório, respostas de EOAET presentes em três das cinco bandas de frequência testadas ${ }^{29,30}$. O critério de passa/falha utilizado por uma pesquisa, cujo objetivo era analisar a amplitude absoluta e nível de resposta das emissões otoacústicas evocadas transientes em recém-nascidos pré-termo e a termo, foi a reprodutibilidade da resposta coclear de, no mínimo, 70\% de correlação e relação sinal-ruído de $6 \mathrm{~dB}$ NPS em 3 frequências, incluindo $4 \mathrm{kHz}{ }^{31}$.

\section{CONCLUSÃO}

As medidas de respostas de EOAET mais robustas (definidas pela relação sinal-ruído SNR) foram observadas para as frequências de 3,2 e $4 \mathrm{kHz}$. Verificou-se que o Protocolo A (4 bandas) apresentou uma taxa de falha elevada, com 20,2\%. Esta porcentagem nos remete a pensar nos casos de falso-positivo, uma vez que podem gerar problemas na qualidade do programa, criando ansiedade desnecessária nos pais, além de aumentar os encaminhamentos ao reteste das falhas das EOAET e sobrecarregar os ambulatórios responsáveis pelo diagnóstico auditivo. No entanto, ambos os Protocolos B (3 bandas; 96,2\%) e C (2 bandas; $96,9 \%)$ não são considerados estatisticamente diferente $(p=0,520)$ na distribuição de passa/ falha, podendo as duas serem utilizadas como protocolos de Programas de TANU.

Apesar dos protocolos $\mathrm{B}$ e $\mathrm{C}$ terem apresentado resultados similares entre os critérios de passa para 2 ou 3 bandas de frequência, deve-se incluir, em estudos futuros, a pesquisa em conjunto com um exame considerado padrão-ouro (Potencial Auditivo de Tronco Encefálico com estímulo clique) visando a confirmar a aplicabilidade clínica destes protocolos de EOAET.Tais achados podem contribuir na escolha de protocolos que tragam maior sensibilidade, especificidade e segurança na realização da triagem auditiva neonatal e possam ser utilizados nas políticas públicas recentemente implantadas no Brasil. 
É importante que o fonoaudiólogo esteja atento a essas questões e, sempre que necessário, busque subsídios os quais denotem evidências que auxiliem quanto à decisão sobre o uso de ferramentas e instrumentos na sua prática clínica. Mas, para que isso ocorra, os centros de estudos e universidades devem contribuir ainda mais fortemente na validação e ampliação de conhecimentos atinentes às aplicações das tecnologias atualmente disponíveis na área da fonoaudiologia.

\begin{abstract}
Purpose: to describe the results of three TEOE protocols obtained from automatic equipment used in a Newborn Hearing Screening Program. Method: during two months, TEOEs of 287 neonates in São Paulo were studied. To register them, we used a three-protocol, set up with different pass/fail criteria. The following protocols were registered: Protocol A: to pass in four frequency bands, not necessarily consecutive-; Protocol B: to pass in three frequency bands, not necessarily consecutive - and Protocol C: to pass in two frequency bands, not necessarily consecutive, as well. The parameters that we used to consider these answers were: reproducibility above $50 \%$, as well as signal/noise ratio $>3 \mathrm{~dB}$ at 1.0 and $1.5 \mathrm{kHz}$ and $>6 \mathrm{~dB}$ at 2.0, 3.0 and $4.0 \mathrm{kHz}$. Results: TEOEs of 574 ears were analyzed and the 2.0, 3.0 and 4.0 frequencies showed the highest pass percentages $(94.1,95.8$ and $92.7 \%$ for protocols $A, B$ and $C$ respectively). Protocol $C$ showed the highest pass percentage, achieving $96,9 \%$. Nevertheless, the results obtained in Protocol B, which had the presence of three bands, showed a similar percentage to Protocol C $(96,2 \%)$. Therefore, there was a not statistically significant difference between Protocols $\mathrm{B}$ and $\mathrm{C}$. $1.0 \mathrm{kHz}$ frequency achieved a percentage of only 9.9 in the tested ears. Conclusions: one has to research Protocols A, B and C with gold standard in order to verify which one suits best in terms of sensibility and specificity, even though the three protocols studied showed similar results in pass criteria for two or three frequency bands.
\end{abstract}

KEYWORDS: Hearing; Hearing Loss; Neonatal Screening; Infant, Newborn

\section{REFERÊNCIAS}

1. Olusanya BO, Wirz BL, Luxon LM. Hospitalbased universal newborn hearing screening for early detection of permanent congenital hearing loss in Lagos, Nigeria. Int J Pediatr otorhinolaryngol. 2008;72:991-1001.

2. Olusanya BO, Luxon LM, Wirz SL. Screening for early childhood hearing loss in Nigeria. J Med Screen. 2005;12: 115-8.

3. Korver AMH, Konings S, Dekker FW, Beers M, Wever CC, Frijns JHM et al. Newborn Hearing Screening vs Later Hearing Screening and Developmental Outcomes in Children With Permanent Childhood Hearing Impairment. JAMA. 2010;304(15):1701-8.

4. Yoshinaga-Itano C, Sedey AL, Coulter DK, Mehl AL. Language of Early- and Later-identified Children With Hearing Loss. Pediatrics. 1998;102(5):1161-71. 5. Bevilacqua MC, Formigoni GMP. Audiologia educacional: uma opção terapêutica para a criança deficiente auditiva. Carapicuíba: Pró-Fono; 1998.

6. Carvalho LS, Cavalheiro LG. Detecção Precoce e Intervenção em Crianças Surdas
Congênitas Inseridas em Escolas Especiais da Cidade de Salvador/BA. Arq. Int. Otorrinolaringol. 2009;13(2):189-94.

7. Sassada MM, Ceccon ME, Navarro JM, Vaz FA. Deficiência auditiva em recém-nascidos internados em unidade de terapia intensiva neonatal. Pediatria. 2005;27(3):163-71.

8. Comitê Brasileiro sobre Perdas Auditivas na Infância (CBPAI) - Recomendação 01/99 [minuta - 2ª versão]. J Cons Fed Fonoaudiol. 2000;5:3-7.

9. Swanepoel D, Ebrahim S, Joseph A, Friedland PL. Newborn hearing screening in a South African private health care hospital. Int $\mathrm{J}$ Pediatr Otorhinolaryngol. 2007;71(6):881-7.

10. Lima GML, Marba ST, Santos MF. Hearing screening in a neonatal intensive care unit. J Pediatr. 2006;82(2):110-4.

11. Lima MCMP, Rossi TRF, Françozo MFC, Marba ST, Lima GML, Santos MFC. Detecção de perdas auditivas em neonatos de um hospital público. Rev. soc. bras. fonoaudiol [periódico na internet]. 2010 [acesso em 2010-10-20];15(1):1-6. Disponível em: http://www.scielo.br/pdf/rsbf/v15n1/03.pdf 
12. Comitê Multiprofissional em Saúde Auditiva (COMUSA). 2009. [acesso em 2010-11-]. Disponível em: http//:www.audiologiabrasil.org.br.

13. Joint Committee on Infant Hearing. Year 2007 Position Statement: principles and guideslines for early hearing detection and intervention programs. Pediatrics. 2007;120(4):898-921.

14. Mcpherson B, Li SF, Shi BX, Tang JL, Wong $B Y$. Neonatal hearing screening: evaluation of toneburst and click-evoked otoacoustic emission test criteria. Ear Hear. 2006;27:256-62.

15. Johnson JL, White KR, Widen JE, Gravel JS, James M, Kennalley $T$ et al. A multicenter evaluation of how many infants with permanent hearing loss pass a two-stage otoacoustic emissions/automated auditory brainstem response newborn hearing screening protocol. Pediatrics. 2005;116(3):663-72. 16. Lin HC, Shu MT, Lee KS, Ho GM, Fu TY, Bruna $S$, et al. Comparison of hearing screening programs between one step with transient evoked otoacoustic emissions (TEOAE) and two steps with TEOAE and automated audtoty brainstem response. Laryngoscope. 2005;115(11):1957-62.

17. Lin $H C$, Shu MT, Lee KS, Lin HY, Lin G. Reducing falser positives in newborn hearing screening program:how and why. Otol Neurotol. 2007;28(6):788-92.

18. Tiensoli LO, Goulart LMH F, Resende LM, Colosino EA. Triagem auditiva em hospital público de Belo Horizonte, Minas Gerais, Brasil: deficiência auditiva e seus fatores de risco em neonatos e lactentes. Cad Saúde Pública. 2007;23(6):1431-41. 19. Benito-Orejas JI, Ramirez B, Morais D, Almaraz A, Fernández-Calvo JL. Comaprasion of two-step transient evoked otoacoustic emissions (TEOAE) and automated auditory brainstem response (AABR) for universal newborn hearing screening programs. Int J Pediatr Otorhinolaryngol. 2008;72(8):1193-201. 20. Granell J, Gavilanes J, Herrero J, Sánches-Jara $\mathrm{JL}$, Velasco MJ, Martin G. Is universal newborn hearing screening more efficient with auditory evoked potentials compared to otoacoustic emissions? Acta Otorrinolaringol Esp. 2008;59(4):170-5.

http://dx.doi.org/10.1590/S1516-18462012005000062

Recebido em: 28/03/2011

Aceito em: 28/07/2011

Endereço para correspondência:

Isabela Freixo Côrtes de Andrade

Avenida Engenheiro Martins Romeo, 103/ 802

Ingá - Niterói - RJ

CEP: 24210-400

E-mail: isabelafono@yahoo.com.br
21. Kemp DT, Bray $P$, Alexander L, Brown AM. Acoustic Emission Cochleography: Pratical aspects. Scand Audiol Suppl. 1986;25:71-95.

22. Probst R. Otoacoustic Emissions: An Overview. Adv Otorhinolgyngol, Basel, Karger. 1990;44:1-91. 23. Pialarissi PR, Gattaz G. Emissões otoacústicas: conceitos básicos e aplicações clínicas. Rev Arquivos da Fundação Otorrinolaringologia. 1997;1(2):13-6.

24. NIH - National Institutes of Health. Consensus Statement - Early identification of hearing loss in infants and young children: Consensus development conference on early identification of hearing loss in infants and young children. NIH. 1993; 11(1):1-3.

25. Sadri M, Thornton AR, Kennedy CR. Effects of maturation on parameters used for pass/fail criteria in neonatal hearing screening programmes using evoked otoacoustic emissions. Audiol Neurootol. 2007;12(4):226-33.

26. Norton SJ, Gorga MP, Widen JE, Folsom RC, Sininger $\mathrm{Y}$, Cone-Wesson $\mathrm{B}$, et al. Identification of neonatal hearing impairment: evaluation of transient evoked otoacoustic emission, distortion product otoacoustic emission, and auditory brain stem response test performance. Ear Hear. 2000; 21:508-28.

27. Popelka GR, Karzon RK, Clary RA. Identification of noise sources that influence distortion product otoacoustic emis sion measurements in human neonates. Ear Hear. 1998;19:319-28.

28. Lopes Fo O, Carlos RC. Emissões Otoacústicas. In: Lopes Fo. O. (ed.) Tratado de Fonoaudiologia. 2a .ed. São Paulo: Tecmedd; 2005. Cap.10.

29. Vohr BR, White KR, Brancia Maxon A, Johnson MJ. Factors affecting the interpretation of transient-evoked otoacousticm emission results in neonatal hearing screening. Semin Hear. 1993; 14:57-72.

30. Brass D, Watkins P, Kemp DT. Assessment of an implementation of a narrow band, neonatal otoacoustic emission screening method. Ear Hear. 1994; 15:467-75.

31. Melo ADPM, Alvarenga KF, Modolo DJ, Bevilacqua MC, Lopes AC, Agostinho-Pesse RS. Emissões otoacústicas evocadas transientes em recém-nascidos a termo e pré-termo. Rev. CEFAC [periódico na internet]. 2010 Feb [acesso em 201102-23];12(1):115-121. Disponível em: http://www. scielo.br/pdf/rcefac/v12n1/a15v12n1.pdf 